\title{
COMMENTS ON "TRANSITION LAYER ON THE SURFACE OF ICE" BY D. BEAGLEHOLE AND D. NASON
}

\author{
N.H. FLETCHER \\ Department of Physics, University of New England, Armidale, NSW 2351, Australia
}

Received 22 September 1981; accepted for publication 15 December 1981

In a recent paper [1] with the title above, Beaglehole and Nason produced elegant evidence, using the techniques of ellipsometry, supporting Faraday"s hypothesis that the surface of ice is covered by a thin equilibrium quasi-liquid layer at temperatures close to the bulk melting point. This evidence ranks with that of Kvlividze et al. [2], using nuclear magnetic resonance techniques on finely powdered ice samples, as among the most direct available and has the advantage that it allows discrimination between the behaviour of surfaces with different crystallographic orientations. The difference in behaviour observed between basal and prism faces may ultimately be able to account for the marked temperature variation of ice crystal habit first documented by Nakaya [3].

In a subsequent note [4] Nason pointed out that the discussion used in the original paper to account for the existence of the liquid layer was in error and would in fact predict a transition region always of monolayer thickness. He proposed that it is necessary to take into account the finite thickness and interaction of the transition layers at the solid-liquid and liquid-vapour interfaces in order to achieve a quasi-liquid layer of finite and temperaturedependent thickness. This he did by adding to the surface free energy a term containing two unknown parameters and varying more or less inversely with the liquid layer thickness.

It is the purpose of the present note to point out that a term of just this type, but containing no unknown parameters, is included in the scmiquantitative theory of the transition layer that I proposed in 1962 [5], a slightly corrected version being given in 1973 [6]. Briefly, the interaction between solid-liquid and liquid-vapour interfaces arises because of molecular orientation at the latter surface caused by the asymmetry of the water molecular dipole (i.e. the existence of quadrupole moments). The bonding coherence in liquid water allows this orientation to decay only exponentially below the surface and produces, in addition, a concentrated double-layer of self-ions $\mathrm{OH}^{-}$and $\mathrm{H}_{3} \mathrm{O}^{+}$, giving a large surface electrical conductivity, as is in fact observed. This theory $[5,6]$ predicts a quasi-liquid layer with an equilibrium 
thickness in nanometres of about

$d \approx(2$ to 5$)-2.5 \log _{10} \Delta T$,

at a temperature $\Delta T$ degrees below the equilibrium melting point. This is of the same general form as the expression deduced from Nason's discussion but rests upon a more explicit set of assumptions. The development leading to (1) did not differentiate between the behaviour of different crystallographic faces, but such a distinction is in fact implicit in the theory if the ice-water interface is treated in more detail [7] and again follows the general direction suggested by Nason. The original discussion [6] also examines the conditions under which the surface film collapses to an oriented monolayer.

An interesting feature of all this discussion is the conclusion that such quasi-liquid layers of appreciable thickness are expected to exist on the surface not only of ice but also of all members of a limited class of materials having asymmetric molecules and exhibiting hydrogen bonding [6]. This conclusion was later verified by photoelectric emission studies $[8,9]$.

Finally I would like to emphasize the fact that a model based upon an essentially water-like film on top of a crystalline ice-like substrate almost inevitably oversimplifies the true situation. Such a model is the easiest approximation from a theoretical standpoint and allows definition of a quantity identifiable as "layer thickness" when experimental results are being considered. It seems most likely, however, that the disordered surface layer is in reality much more structured than is bulk water and its detailed properties certainly vary continuously across its thickness.

\section{References}

[1] D. Beaglehole and D. Nason, Surface Sci. 96 (1980) 357.

[2] V.I. Kvlividze, V.F. Kiselev and L.A. Ushakova, Dokl. Akad. Nauk USSR, Ser. Fiz. Khim. $191(1970) 307$.

[3] U. Nakaya, Snow Crystals: Natural and Artificial (Harvard University Press, Cambridge, MA, 1954).

[4] D. Nason, Surface Sci. 102 (1981) L7.

[5] N.H. Fletcher, Phil. Mag. 7 (1962) 255; 8 (1963) 1425; 18 (1968) 1287.

[6] N.H. Fletcher, in: Physics and Chemistry of Ice, Eds. E. Whalley, S.J. Jones and L.W. Gold (Royal Society of Canada, Ottawa, 1973).

[7] N.H. Fletcher, J. Crystal Growth 28 (1975) $375 ; 35$ (1976) 39.

[8] D. Nason and N.H. Fletcher, J. Chem. Phys. 62 (1975) 4444.

[9] D. Nason, J. Chem. Phys. 64 (1976) 3930. 